\title{
Baseline Predictors of Health-Related Quality of Life After Anterior Cruciate Ligament Reconstruction
}

\author{
A Longitudinal Analysis of a Multicenter Cohort at Two and Six Years \\ Warren R. Dunn, MD, MPH, Brian R. Wolf, MD, MS, Frank E. Harrell Jr., PhD, Emily K. Reinke, PhD, \\ Laura J. Huston, MS, MOON Knee Group*, and Kurt P. Spindler, MD
}

\begin{abstract}
Background: Limited information exists regarding predictors of general quality of life following anterior cruciate ligament $(A C L)$ reconstruction with up to six-year follow-up. We hypothesized that certain variables evaluated at the time of $\mathrm{ACL}$ reconstruction will predict the general quality of life as measured by the Short Form-36 (SF-36).
\end{abstract}

Methods: All unilateral ACL reconstructions from 2002 to 2004 in patients currently enrolled in a prospective multicenter cohort were evaluated. Patients preoperatively completed the SF-36 validated outcome instrument. Surgeons documented intra-articular pathological conditions and treatment, as well as the ACL reconstruction surgical technique. At baseline and at a minimum of two and six years postoperatively, patients completed the SF-36. Longitudinal analysis was performed for the two-year and six-year end points.

Results: Of the initial 1512 subjects, at least one follow-up questionnaire was obtained from 1411 subjects (93\%). The cohort was $44 \%$ female, and the median patient age at enrollment was twenty-three years. The mean scores were 41.9 points for the Physical Component Summary (PCS) and 51.7 points for the Mental Component Summary (MCS) at baseline, 53.6 points for the PCS and 52.0 points for the MCS at two years, and 54.0 points for the PCS and 52.4 points for the MCS at six years. Significant predictors of a higher PCS score were a higher baseline PCS score, younger age, lower baseline body mass index, having $>50 \%$ of the lateral meniscus excised, or having no treatment done on a lateral meniscal tear. In contrast, significant predictors of a lower PCS score were a shorter follow-up time since surgery, revision ACL reconstruction, smoking at baseline, fewer years of education, and chondromalacia of the lateral tibial plateau. The mean utility gained at six years after ACL reconstruction was 5.3 quality-adjusted life years (QALYS).

Conclusions: Large improvements in the PCS (with an effect size of 1.2) were noted at two years and were maintained at six years after ACL reconstruction. Lower education and smoking were significant predictors of lower PCS and MCS scores. ACL reconstruction resulted in a relatively high gain of QALYS.

Level of Evidence: Prognostic Level I. See Instructions for Authors for a complete description of levels of evidence.

Peer Review: This article was reviewed by the Editor-in-Chief and one Deputy Editor, and it underwent blinded review by two or more outside experts. It was also reviewed by an expert in methodology and statistics. The Deputy Editor reviewed each revision of the article, and it underwent a final review by the Editor-in-Chief prior to publication. Final corrections and clarifications occurred during one or more exchanges between the author(s) and copyeditors.

*Samuel K. Nwosu, MS, Christopher C. Kaeding, MD, Richard D. Parker, MD, Rick W. Wright, MD, Jack T. Andrish, MD, Eric C. McCarty, MD, Annunziato Amendola, MD, Robert G. Marx, MD, MSc, Michelle L. Wolcott, MD, Zhouwen Liu, MS, and JoAnn M. Alvarez, MS, are MOON (Multicenter Orthopaedic Outcomes Network) Knee Group members.

Disclosure: One or more of the authors received payments or services, either directly or indirectly (i.e., via his or her institution), from a third party in support of an aspect of this work. In addition, one or more of the authors, or his or her institution, has had a financial relationship, in the thirty-six months prior to submission of this work, with an entity in the biomedical arena that could be perceived to influence or have the potential to influence what is written in this work. Also, one or more of the authors has a patent or patents, planned, pending, or issued, that is broadly relevant to the work. Finally, one or more of the authors has had another relationship, or has engaged in another activity, that could be perceived to influence or have the potential to influence what is written in this work. The complete Disclosures of Potential Conflicts of Interest submitted by authors are always provided with the online version of the article.

Disclaimer: The contents of this article are solely the responsibility of the authors and do not necessarily represent official views of the National Center for Advancing Translational Sciences or the National Institutes of Health. 
The Journal of Bone \& Joint Surgery $\cdot$ Jbjs.org VOlume 97-A • Number 7 - April 1, 2015
Baseline Predictors of Health-Related Quality of Life After Anterior Cruciate Ligament Reconstruction
$\mathrm{T}$ here is sparse literature regarding the prognosis and predictors of anterior cruciate ligament (ACL) reconstruction outcomes at six years as measured by validated patient-based outcome instruments and assessed by multivariable analysis. Knowing prognostic information would be valuable in physician counseling of patients considering ACL reconstruction.

Large sample sizes with adequate follow-up are necessary for such analysis. A previously published randomized controlled trial had an enrollment of 225 patients ${ }^{1}$, which limits risk factor analysis. A previous cohort study utilizing multivariable analysis was limited by $69 \%$ follow-up and the lack of baseline measurements of the outcomes ${ }^{2}$, which are important to adjust for, as they are often the strongest predictor of follow-up scores.

The Short Form-36 (SF-36) is a widely used measure of general quality of life. It allows for comparison across different disease categories; therefore, normative-based scoring converts the scores such that they have direct interpretation related to the general United States population, with a mean score (and standard deviation) of $50 \pm 10$ points. Hence, a 1-point change in a score is one-tenth of a standard deviation or an effect size of $0.10^{3}$. The Short Form- 6 dimension (SF-6D), a preferencebased utility measure, can be calculated from the SF-36, allowing the calculation of quality-adjusted life years (QALYs) ${ }^{4}$. Previous studies utilizing the SF-36 in athletic populations have compared domain scores, ranging from 0 to 100 points, to normative values, which do not as easily lend themselves to interpretation, but have not reported normative-based scores ${ }^{5,6}$. The eight subscales of the SF-36 can be collapsed into two components, the Physical Component Summary (PCS) score and the Mental Component Summary (MCS) score. The use of the PCS and MCS has been advocated for large studies, particularly when there is a focus on the general effect on health?

The aim of the current study was to determine the prognosis and predictors of health-related quality of life as measured by the SF-36 at two and six years after surgery as well as the utility gained following ACL reconstruction as measured by the SF-6D. These results should aid evidence-based decision-making as related to a patient's prognosis following ACL reconstruction, should provide a high level of evidence for surgeon decisionmaking, and should have the potential to identify future modifiable risk factors that could be altered to improve outcomes of ACL reconstruction. Furthermore, the improvements in health-related quality of life and utility according to the SF-6D should provide justification for expenditures related to patients with ACL injuries.

\section{Materials and Methods Study Population}

The Multicenter Orthopaedic Outcomes Network (MOON) Knee Group began on January 1, 2002, as a consortium of six sites with eight surgeons with the aim of prospectively enrolling and following subjects undergoing ACL reconstruction. Vanderbilt University served as the data-coordinating center for the study and was responsible for entering baseline data and for collecting follow-up data on all subjects. Institutional review board approval was obtained from all participating centers. All subjects who had undergone ACL reconstruction at participating sites in 2002 through 2004 (from January 1, 2002, to December 31, 2004) were invited to enroll in the study (Fig. 1).

\section{Data Sources}

Following documentation of written informed consent, participants completed a thirteen-page questionnaire that has been described previously ${ }^{8}$. Regarding the thirteen-page questionnaire, the SF-36 was used as a measure of general health. The SF-6D can be calculated from the SF-36, allowing the calculation of QALYs ${ }^{4}$. This questionnaire was completed before the day of surgery in the vast majority of cases; otherwise, it was completed within two weeks following the surgery date. If it was completed following surgery, subjects were instructed to complete it from the preoperative perspective.

Surgeons completed a forty-nine-page questionnaire that has been described previously ${ }^{9}$. Meniscal injuries were classified by size, location, and type of tear (partial or complete), and treatment was recorded as not treated, repaired, or the extent of resection ${ }^{10}$. All patients were given standardized evidence-based guidelines for ACL reconstruction rehabilitation ${ }^{11,12}$.

\section{Study End Points}

The eight SF-36 domain scores and the two component summary scores were measured longitudinally at follow-ups of at least two and six years. The PCS and MCS were the primary end points used for multivariable modeling. Subjects could contribute either two-year or six-year end points or both. Lastly, the utility gained at six years using the SF-6D was calculated.

\section{Statistical Analysis}

Multivariable analysis is necessary to see not only how different factors influence outcome but also what the relative strength of the association is. In evaluating the ACL-injured knee, associated meniscal tears and articular cartilage injury cannot be randomized. Therefore, a prospective cohort is the preferred design. Further, because these injuries are often induced at the same time as the ACL injury, a natural experiment has occurred given that a proportion of the ACL injuries will have concomitant intra-articular damage and others will not. In cohort studies, where variables cannot be randomly and equally distributed, multivariable analysis is the only way to account for uneven distributions of factors and potential confounders ${ }^{13}$. Risk factors likely to be relevant to ACL reconstruction outcomes include age, sex, mechanism of injury, body mass index (BMI), concomitant medial and lateral meniscal tears and treatment, articular cartilage injuries and treatment, ACL reconstruction technique, and graft choice ${ }^{14}$.

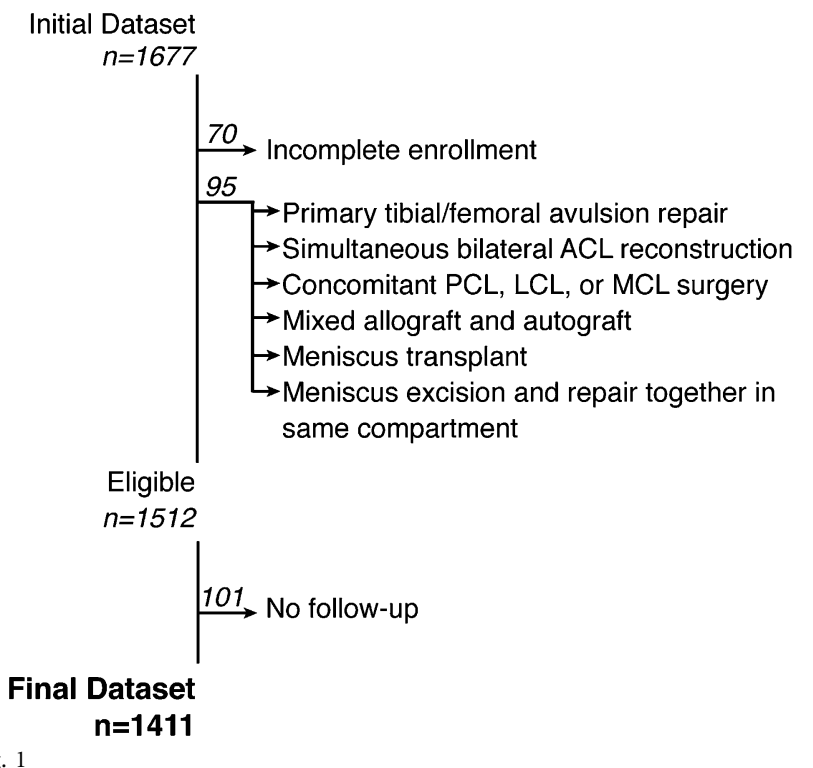

Flow diagram of subject enrollment. $\mathrm{PCL}=$ posterior cruciate ligament, $\mathrm{LCL}=$ lateral cruciate ligament, and $\mathrm{MCL}=$ medial cruciate ligament. 
The Journal of Bone \& Joint Surgery $\cdot$ Jbjs.org Volume 97-A • Number 7 · April 1, 2015
Baseline Predictors of Health-Related Quality of Life After Anterior Cruciate Ligament Reconstruction
Multivariable analysis was used to determine which baseline variables measured at the time of the index ACL surgery were significant predictors of healthrelated quality of life at two and six years after surgery. Longitudinal analysis was performed with use of proportional-odds ordinal logistic regression to fit a single model for the two-year and six-year end points ${ }^{15}$. We used the Huber-White cluster sandwich estimator to adjust the variance-covariance matrices to correct for correlated responses from two observations on the same patient ${ }^{16}$.

The proportional-odds model makes fewer distributional assumptions than ordinary regression. The subscales of the SF-36 as well as the component summary scores were the dependent variables, and one model was fit for each. The independent covariates in the models included the baseline measure of the outcome, patient age, sex, race, education level, smoking status, BMI, activity level as assessed with use of the Marx activity rating scale ${ }^{17}$, the patient-reported primary sport and competition level, the surgeon's years of experience, the year of surgery, the follow-up time since surgery, whether the patient had a previous ACL reconstruction on the contralateral knee, whether the surgery was a revision, graft type, degree of damage to the cartilage, meniscal pathological condition and treatment, and whether the hoop stress fibers of either meniscus were disrupted. Missing data were imputed and continuous covariates (e.g., age) were modeled with use of splines to permit nonlinear relationships (see Appendix).

Nomograms were constructed to display the relationships between predictor variables and the outcomes, based on the fitted models. A nomogram can be used to estimate the mean response for individual patients and to show the relationship among the different predictor variables and how this affects the response.
Statistical analysis was performed with free open-source R statistical software and the rms package ${ }^{18,19}$.

\section{Source of Funding}

This project was partially funded by grants from the National Institutes of Health/National Institute of Arthritis and Musculoskeletal and Skin Diseases (5K23 AR052392 and 5R01 AR053684). This project was partially supported by the Clinical \& Translational Science Awards (award number UL1TR000445) from the National Center for Advancing Translational Sciences. The project was also supported by the Vanderbilt Sports Medicine Research Fund, which received unrestricted educational gifts from Smith \& Nephew Endoscopy and DonJoy Orthopaedics. Funds were used to pay for salaries, core biostatistical support, subject remuneration, and study supplies.

\section{Results}

\section{Study Population}

Crom January 1, 2002, to December 31, 2004, 1512 subjects met the inclusion criteria of having a unilateral ACL reconstruction and are included in our analyses; see the flow diagram for exclusion criteria (Fig. 1). Of the initial 1512 subjects, at least one repeat questionnaire was obtained from 1411 subjects (93\%). Two-year follow-up was obtained for 1308 subjects (87\%), and six-year follow-up was obtained for

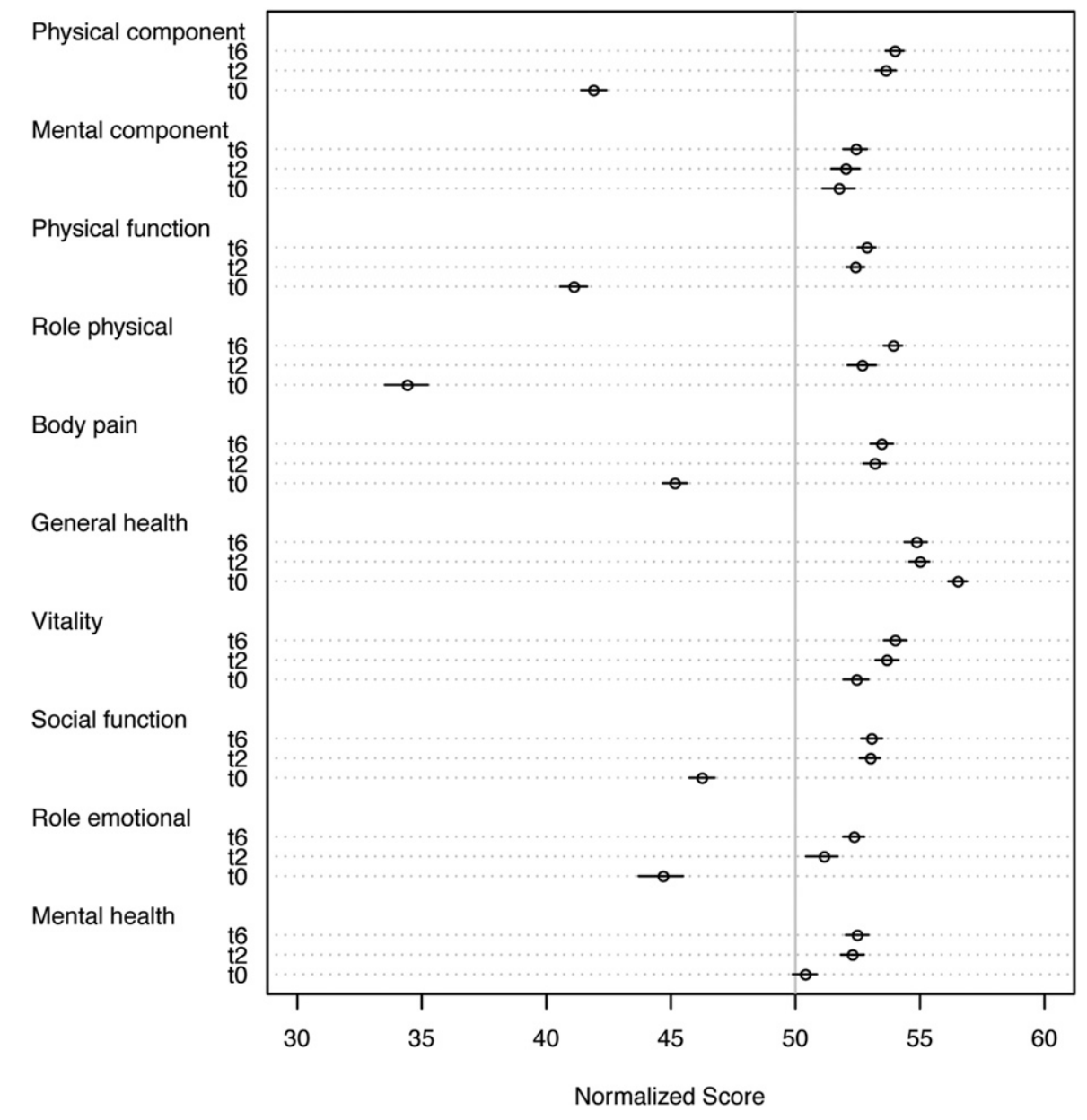

Fig. 2

Normative-based scoring of the SF-36 profile. t0 = baseline, t2 = two-year follow-up, and t6 = six-year follow-up. 
Baseline Predictors of Health-Related Quality of Life After anterior Cruciate Ligament Reconstruction

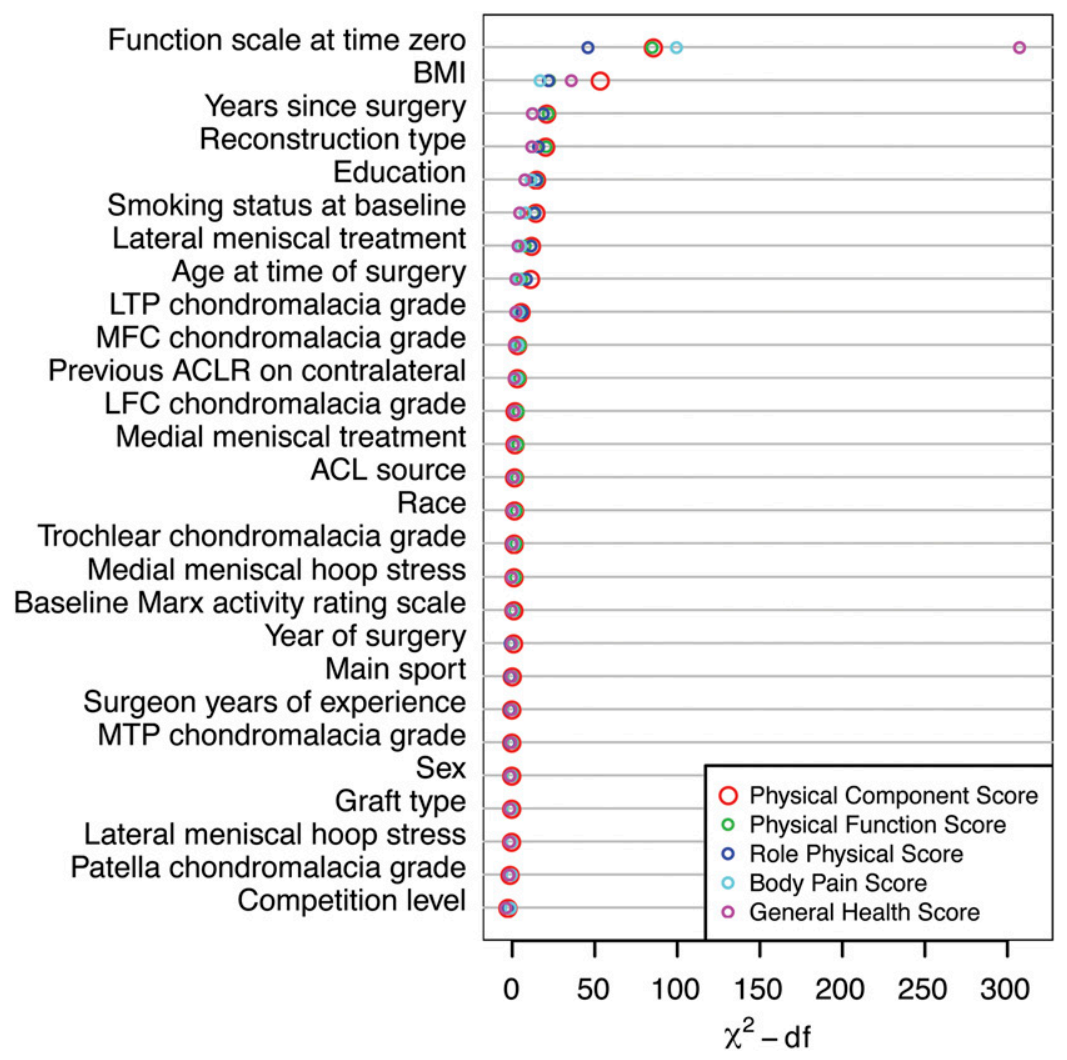

Fig. 3

Relative proportion of variance accounted for by each term, combined across physical domains. LTP = lateral tibial plateau, MFC = medial femoral condyle, $A C L R=A C L$ reconstruction, $L F C=$ lateral femoral condyle, MTP $=$ medial tibial plateau, and $d f=$ degrees of freedom.

1307 subjects (86\%). The median age of the cohort was twentythree years (interquartile range [IQR], seventeen to thirty-five years), and the cohort was $44 \%$ female.

\section{Study End Points}

The mean subscale scores as well as the PCS and MCS scores at baseline and two and six years are presented in Figure 2. The mean scores were 41.9 points for the PCS and 51.7 points for the MCS at baseline, 53.6 points for the PCS and 52.0 points for the MCS at two years, and 54.0 points for the PCS and 52.4 points for the MCS at six years. The mean utility gained at six years was 5.3 QALYs.

Multivariable analysis was used to determine which baseline variables measured at the time of index ACL surgery were significant predictors of health-related quality of life at two and six years after surgery. We summarized the results of the ten models by including two plots, one for the physical domain models and the PCS (Fig. 3) and one for the mental domain models and the MCS (Fig. 4). The plots show the independent variables on the vertical axis, and the relative portion of the variation in the outcome accounted for by the given variable on the horizontal axis. This importance is measured by Wald chi-square statistics minus the degrees of freedom. The subscale measured at the time of surgery tended to be the most important variable in predicting the corresponding subscale measured at subsequent times. The different domain scores showed consistency in the ranking of the importance of the independent variables. Hence, results are presented for the two summary scores and not for all subscale scores.

\section{PCS}

The following variables were significant predictors of higher scores: a higher baseline PCS (IQR odds ratio [the change in log odds from the twenty-fifth to the seventy-fifth percentile for continuous predictors], 1.57 [95\% confidence interval $\{95 \% \mathrm{CI}\}$, 1.26 to 1.96$] ; \mathrm{p}<0.0001$ ); younger age (IQR odds ratio, 2.04 [95\% CI, 1.28 to 3.23]; $\mathrm{p}=0.002$ ); lower baseline BMI (IQR odds ratio, 1.35 [95\% CI, 1.18 to 1.53 ]; $\mathrm{p}<0.0001$ ); lateral meniscal treatment $(\mathrm{p}=0.009)$, specifically, having $>50 \%$ of the lateral meniscus excised (IQR odds ratio, 2.45 [95\% CI, 1.49 to 4.01]); or having no treatment done for a lateral meniscal tear (IQR odds ratio, 1.27 [95\% CI, 1.01 to 1.59$]$ ).

The following variables were significant predictors of lower scores: shorter follow-up time since surgery (IQR odds ratio, 0.64 [ $95 \% \mathrm{CI}, 0.50$ to 0.82 ]; $\mathrm{p}<0.0001$ ), revision ACL reconstruction (IQR odds ratio, 0.51 [95\% CI, 0.39 to 0.68]; $\mathrm{p}<0.0001$ ), fewer years of education (IQR odds ratio, 0.70 [ $95 \% \mathrm{CI}, 0.59$ to 0.84 ]; $\mathrm{p}=0.0001$ ), current smoker (IQR odds ratio, 0.52 [95\% CI, 0.37 to 0.73 ]; $\mathrm{p}=0.0004)$, and chondromalacia of the lateral tibial plateau (IQR odds ratio, 0.53 [95\% CI, 0.31 to 0.92 ]; $\mathrm{p}=0.03$ ).

A nomogram, which can be used to estimate the mean response for individual patients and to show the relationship 
The Journal of Bone \& Joint Surgery $\cdot$ JBjs.org VOlume 97-A • Number 7 - April 1, 2015
Baseline Predictors of Health-Related Quality of Life After Anterior Cruciate Ligament Reconstruction

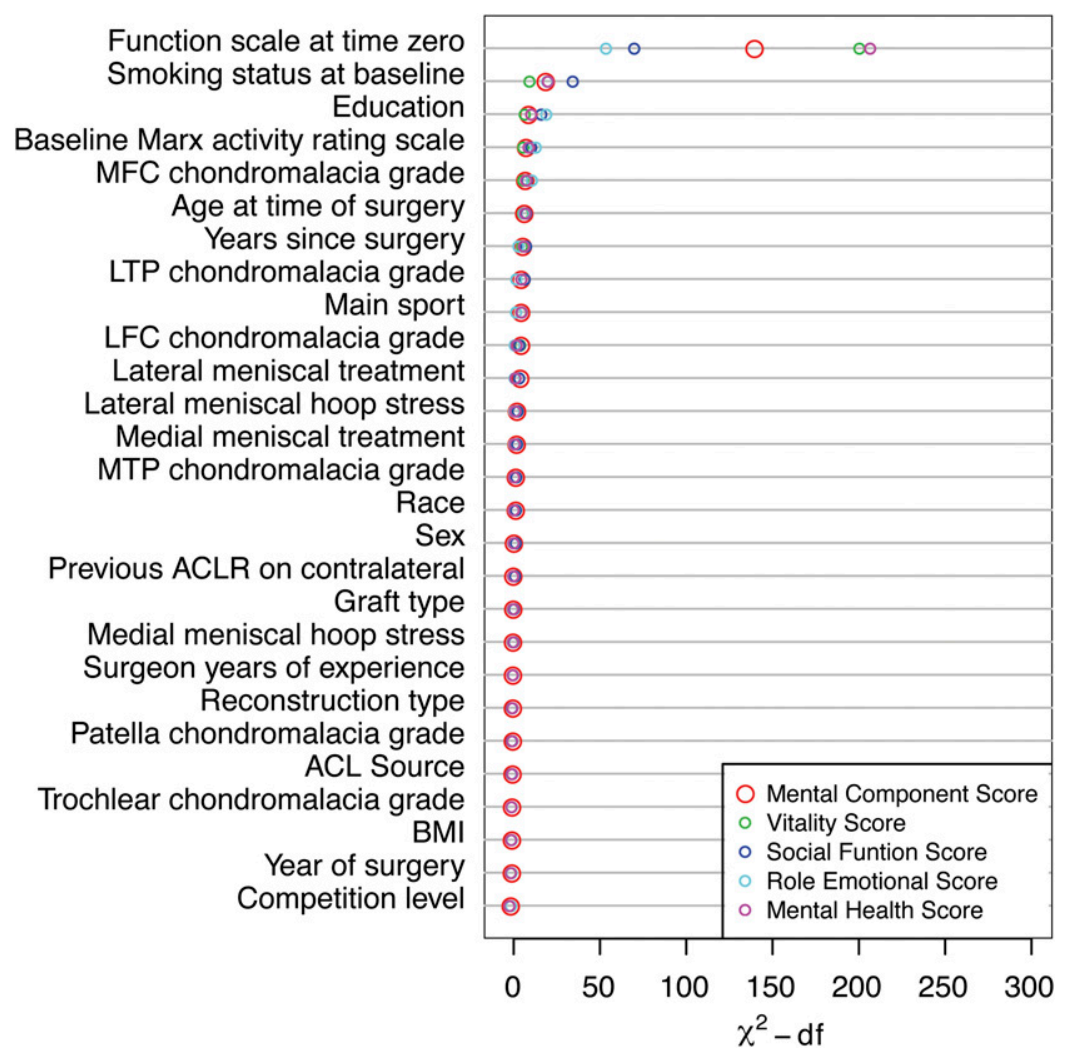

Fig. 4

Relative proportion of variance accounted for by each term, combined across mental domains. MFC $=$ medial femoral condyle, LTP $=$ lateral tibial plateau, $\mathrm{LFC}=$ lateral femoral condyle, MTP $=$ medial tibial plateau, $\mathrm{ACLR}=\mathrm{ACL}$ reconstruction, and df $=$ degrees of freedom.

among the different predictor variables and how this affects the predicted PCS score, is shown in the Appendix.

\section{MCS}

The significant predictors of a higher MCS were a higher baseline MCS (IQR odds ratio, 1.99 [95\% CI, 1.58 to 2.52]; $\mathrm{p}<0.0001$ ) and a higher baseline Marx activity rating scale score (IQR odds ratio, 1.46 [95\% CI, 1.13 to 1.88]; $\mathrm{p}=0.01$ ). The following variables were significant predictors of a lower MCS: fewer years of education (IQR odds ratio, 0.76 [95\% CI, 0.64 to 0.92]; $\mathrm{p}=$ $0.003)$ and smoking status $(\mathrm{p}<0.0001)$, specifically, being a previous smoker (IQR odds ratio, 0.72 [95\% CI, 0.52 to 0.99$]$ ) or current smoker (IQR odds ratio, 0.50 [95\% CI, 0.36 to 0.69]). A nomogram of the MCS model is shown in the Appendix.

\section{Discussion}

This study produced several interesting findings that merit further discussion. First, this study showed that the SF-36 is responsive to ACL reconstruction. Next, although no significant changes were observed in the MCS in this relatively young and healthy cohort, large improvements were noted in the PCS at two years and were maintained at six years (effect size of 1.2 at two and six years), demonstrating the durability of the improvement after ACL reconstruction. Specific factors were identified as significant predictors of PCS scores and MCS scores. Our data demonstrated gained QALYs after ACL recon- struction and this gain was clinically important. Lastly, our data exposed some perplexing relationships between concomitant meniscal surgery and SF-36 outcomes after ACL reconstruction.

Our study demonstrated that ACL reconstruction resulted in large improvements in the PCS, with a mean improvement of 12 points at both the two-year and six-year follow-ups. It was encouraging to see maintenance of improved physical scores up to six years after surgery, which demonstrates intermediate-term durability. Baseline activity level as measured with use of the Marx scale was a significant predictor of MCS scores but not PCS scores in the current study. To our knowledge, comparative literature has been limited. Eitzen et al. found lower bodily pain subscores at two years in subjects with concomitant ACL reconstruction and meniscal injuries requiring treatment, but subjects who had meniscal injuries requiring repair were excluded from that study ${ }^{20}$. Månsson et al. examined baseline predictors of health-related quality of life using the SF- 36 at three to six years following ACL reconstruction and found that the pre-injury activity level was a predictor of two of the SF-36 subscales $^{21}$. Specifically, a higher baseline Tegner activity level was associated with higher general health scores and lower role emotional scores, whereas we did not find activity level to predict outcome. This discrepancy may be due to the different activity scales (Marx compared with Tegner) used in the two studies.

In contrast to the physical score changes after ACL reconstruction, the MCS and the general health subscale were 
The Journal of Bone \& Joint Surgery $\cdot$ Jbjs.org VOlume 97-A • Number 7 - April 1, 2015
Baseline Predictors of Health-Related Quality of Life After Anterior Cruciate Ligament Reconstruction both well above the population norm of 50 points at baseline and did not change dramatically over time (Fig. 2). The subjects also reported baseline mental health and vitality subscale scores above the population norm of 50 points (Fig. 2). This reflects the relatively young and healthy patient cohort studied. Consistent with our data, Busija et al. found the SF-36 to be a useful metric for measuring group changes over time for several orthopaedic procedures, including ACL reconstruction, and noted that there had been very little change in the general health subscale over the two-year follow-up period ${ }^{22}$. However, others have noted that the baseline subscale scores, excluding general health, have been below population norms prior to ACL reconstruction ${ }^{22,23}$. Although subjects in the current study had baseline general health, vitality, and mental health subscale scores above the population norm of 50 points, the other five subscale scores were below the norm prior to surgery.

The QALY findings are intriguing and novel for ACL reconstruction. One QALY represents one year of perfect health. Six months of perfect health, or one year of health that is considered to be half as good as perfect health, corresponds to 0.5 QALY.

The mean utility gained at six years in the current study was 5.3 QALYs. To our knowledge, five authors have previously reported on QALYs gained following ACL reconstruction, and these have ranged from 0.78 to $5.1^{24-28}$. QALYs have been reported following other surgical procedures as well. Vitale et al. found that the mean QALYs gained at one year following rotator cuff repair were 0.81 using the Health Utility Index and 3.43 using the European Quality-of-Life measure ${ }^{29}$. Räsänen et al. found that the mean QALYs gained at one year were 1.3 for primary total hip arthroplasty, 0.2 for revision total hip arthroplasty, and 0.6 for primary total knee arthroplasty ${ }^{30}$. Al-Ruzzeh et al. found that the mean QALY gained at six months following coronary artery bypass surgery was $0.3^{31}$. Thus, the gain in QALYs after ACL reconstruction exceeds other procedures renowned for improving quality of life. It appears that health-care expenditures on ACL reconstruction are clearly justified by these findings.

The outcomes relative to meniscal treatment in the setting of ACL reconstruction are perplexing. However, in the current cohort of more than 1400 ACL reconstruction cases, meniscal treatment is not easily dismissed. Our data demonstrated that having $>50 \%$ of the lateral meniscus excised was associated with higher outcome scores. This may be the most difficult finding to explain. With an ACL tear, the knee pivots and the anterolateral aspect of the distal part of the femur contacts the posterior aspect of the lateral tibial plateau. In some cases, this can be quite traumatic and can result in subclinical impaction fractures noted as bone bruising on magnetic resonance imaging. Lateral meniscal tears are commonly seen at the time of acute ACL injury, and this is thought to be due to the meniscus being trapped or crushed during these pivoting events. In the MOON cohort, lateral meniscal tears were not found in $49 \%$ of ACL reconstruction cases. One could theorize that the meniscus is not normal (despite the absence of a tear), or is covertly damaged, in those cases in which resection did not occur, resulting in lower outcomes. The relationship between meniscectomy and meniscal repair after ACL recon- struction is compelling enough that it warrants further investigation. Our data would suggest that current meniscal tear treatments and algorithms need to be closely examined and to be investigated further. We do not advocate resection of a normalappearing meniscus. These intermediate-term data also cannot speak to possible long-term outcomes relative to meniscuspreserving compared with meniscus-removing surgery in the setting of ACL reconstruction. Leaving some lateral meniscal tears untreated was associated with improved PCS scores, consistent with previous studies ${ }^{9,32}$.

This study had several limitations. The SF-36 is a general health measure and may not be as responsive to ACL reconstruction as joint and condition-specific measures. These knee and disease-specific data were collected but were beyond the scope of the current study, in which impact on general health was the focus. It is possible that other potential influential variables were not included in our analysis efforts despite our attempt to use an exhaustive list of potential variables. To our knowledge, there has been limited information in the orthopaedic literature regarding QALYs, and thus the relative importance of the QALYs gained after ACL reconstruction is difficult to relate in the context of other procedures. Lastly, the findings related to meniscal treatment are perplexing. It is possible that these findings do not reflect reality. However, intensive scrutiny and the use of a very large cohort size suggest that there is merit to further investigating the role of meniscal treatment on outcome in the setting of ACL reconstruction.

In summary, ACL reconstruction results in a large improvement in the SF-36 PCS at two years and is durable to a minimum of six years. The QALYs gained after ACL reconstruction are substantial relative to other successful operations where this has been explored, suggesting that ACL reconstruction utilization is appropriate. Lastly, interesting relationships appear to exist between the treatment of meniscal pathological conditions during concomitant ACL reconstruction and the ultimate outcome assessed using SF-36 measures, which may be explained by patient characteristic confounders.

\section{Appendix}

eA Text providing the details of the statistical methods, figeA ures showing nomograms for the SF-36 PCS and MCS score models, and tables showing the baseline and follow-up cohort descriptive statistics and the modeled data descriptive statistics are available with the online version of this article as a data supplement at jbjs.org.

NoTE: The authors thank the following research coordinators, analysts, and support staff from the Multicenter Orthopaedic Outcomes Network (MOON) sites, whose efforts make this consortium possible: Kristen Banjac, Breanna Beck, Lynn Borzi, Julia Brasfield, Maxine Cox, Lisa Hegemier, Michelle Hines, and Leah Schmitz from the Cleveland Clinic; Angela Pedroza, Kari Stammen, and Rose Backs from The Ohio State University; Carla Britton, Catherine Fruehling-Wall, and Patty Stolley from the University of lowa; Christine Bennett, John Gines, and Paula Langner from the University of Colorado; Linda Burnworth, Robyn Gornati, and Amanda Haas from Washington University in St. Louis; Brian Boyle, Patrick Grimm, Kaitlynn Lillemoe, and Lana Verkuil from the Hospital for Special Surgery; and Thomas Dupont, John Shaw, Suzet Galindo-Martinez, Angela Keen, Erica Scaramuzza, Morgan Crawford, and Lynn Cain from Vanderbilt University.

Warren R. Dunn, MD, MPH

University of Wisconsin Medical Center, 
The Journal of Bone \& Joint Surgery $\cdot$ Jbjs.org VOlume 97-A • Number 7 - April 1, 2015
Baseline Predictors of Health-Related Quality of Life After Anterior Cruciate Ligament Reconstruction
Centennial Building,

1685 Highland Avenue,

Madison, WI 53705

Brian R. Wolf, MD, MS

University of Iowa Sports Medicine Center,

2701 Prairie Meadow Drive,

Iowa City, IA 52242

Frank E. Harrell Jr., PhD

Department of Biostatistics,

Vanderbilt University Medical Center,

Suite 11000, 2525 West End Avenue,

Nashville, TN 37203-1738
Emily K. Reinke, PhD

Laura J. Huston, MS

MOON Knee Group*

Department of Orthopaedic Surgery and Rehabilitation,

Vanderbilt University Medical Center,

4200 Medical Center East, South Tower,

Nashville, TN 37232-8774

Kurt P. Spindler, MD

Cleveland Clinic,

Sports Medicine A-41,

9500 Euclid Avenue,

Cleveland, OH 44195.

E-mail address: spindlk@ccf.org

\section{References}

1. O'Neill DB. Arthroscopically assisted reconstruction of the anterior cruciate ligament. A follow-up report. J Bone Joint Surg Am. 2001 Sep;83(9):1329-32.

2. Spindler KP, Warren TA, Callison JC Jr, Secic M, Fleisch SB, Wright RW. Clinical outcome at a minimum of five years after reconstruction of the anterior cruciate ligament. J Bone Joint Surg Am. 2005 Aug;87(8):1673-9.

3. Ware J, Kosinski M, Dewey JE. How to score version 2 of the SF-36 health survey. Lincoln, Rl: QualityMetric Incorporated; 2000.

4. Brazier J, Roberts J, Deverill M. The estimation of a preference-based measure of health from the SF-36. J Health Econ. 2002 Mar;21(2):271-92.

5. Huffman GR, Park J, Roser-Jones C, Sennett BJ, Yagnik G, Webner D. Normative SF-36 values in competing NCAA intercollegiate athletes differ from values in the general population. J Bone Joint Surg Am. 2008 Mar;90(3):471-6.

6. McAllister DR, Motamedi AR, Hame SL, Shapiro MS, Dorey FJ. Quality of life assessment in elite collegiate athletes. Am J Sports Med. 2001 Nov-Dec;29(6):806-10.

7. Patel AA, Donegan D, Albert T. The 36-item short form. J Am Acad Orthop Surg. 2007 Feb;15(2):126-34.

8. Dunn WR, Spindler KP; MOON Consortium. Predictors of activity level 2 years after anterior cruciate ligament reconstruction (ACLR): a Multicenter Orthopaedic Outcomes Network (MOON) ACLR cohort study. Am J Sports Med. 2010 Oct; 38(10):2040-50. Epub 2010 Aug 13.

9. Spindler KP, Huston LJ, Wright RW, Kaeding CC, Marx RG, Amendola A, Parker RD, Andrish JT, Reinke EK, Harrell FE Jr, Dunn WR; MOON Group. The prognosis and predictors of sports function and activity at minimum 6 years after anterior cruciate ligament reconstruction: a population cohort study. Am J Sports Med. 2011 Feb; 39(2):348-59. Epub 2010 Nov 17.

10. Dunn WR, Wolf BR, Amendola A, Andrish JT, Kaeding C, Marx RG, McCarty EC, Parker RD, Wright RW, Spindler KP. Multirater agreement of arthroscopic meniscal lesions. Am J Sports Med. 2004 Dec;32(8):1937-40.

11. Wright RW, Preston E, Fleming BC, Amendola A, Andrish JT, Bergfeld JA, Dunn WR, Kaeding C, Kuhn JE, Marx RG, McCarty EC, Parker RC, Spindler KP, Wolcott M, Wolf $\mathrm{BR}$, Williams GN. A systematic review of anterior cruciate ligament reconstruction rehabilitation: part I: continuous passive motion, early weight bearing, postoperative bracing, and home-based rehabilitation. J Knee Surg. 2008 Jul;21(3):217-24.

12. Wright RW, Preston E, Fleming BC, Amendola A, Andrish JT, Bergfeld JA, Dunn WR, Kaeding C, Kuhn JE, Marx RG, McCarty EC, Parker RC, Spindler KP, Wolcott M, Wolf BR, Williams GN. A systematic review of anterior cruciate ligament reconstruction rehabilitation: part II: open versus closed kinetic chain exercises, neuromuscular electrical stimulation, accelerated rehabilitation, and miscellaneous topics. J Knee Surg. 2008 Jul;21(3):225-34.

13. Katz MH. Multivariable analysis: a primer for readers of medical research. Ann Intern Med. 2003 Apr 15;138(8):644-50.

14. Kaeding CC, Aros B, Pedroza A, Pifel E, Amendola A, Andrish JT, Dunn WR, Marx RG, McCarty EC, Parker RD, Wright RW, Spindler KP. Allograft versus autograft anterior cruciate ligament reconstruction: predictors of failure from a MOON prospective longitudinal cohort. Sports Health. 2011 Jan;3(1):73-81.

15. Walker SH, Duncan DB. Estimation of the probability of an event as a function of several independent variables. Biometrika. 1967 Jun;54(1):167-79.

16. Lin DY. Cox regression analysis of multivariate failure time data: the marginal approach. Stat Med. 1994 Nov 15;13(21):2233-47.

17. Marx RG, Stump TJ, Jones EC, Wickiewicz TL, Warren RF. Development and evaluation of an activity rating scale for disorders of the knee. AM J Sports Med. 2001 Mar-Apr;29(2):213-8.
18. $R$ Development Core Team. The $R$ project for Statistical Computing. Vienna: $R$ Foundation for Statistical Computing; 2010. http://www.R-project.org/. Accessed 2014 Dec 8.

19. Harrell FE Jr. RMS: regression modeling strategies. 2011. http://CRAN.Rproject.org/package $=$ rms. Accessed 2014 Dec 8.

20. Eitzen I, Holm I, Risberg MA. Preoperative quadriceps strength is a significant predictor of knee function two years after anterior cruciate ligament reconstruction. Br J Sports Med. 2009 May;43(5):371-6. Epub 2009 Feb 17.

21. Månsson 0 , Kartus $\mathrm{J}$, Sernert N. Pre-operative factors predicting good outcome in terms of health-related quality of life after ACL reconstruction. Scand J Med Sci Sports. 2013 Feb;23(1):15-22. Epub 2012 Jan 31.

22. Busija L, Osborne RH, Nilsdotter A, Buchbinder R, Roos EM. Magnitude and meaningfulness of change in SF-36 scores in four types of orthopedic surgery. Health Qual Life Outcomes. 2008 Jul 31;6:55.

23. Shapiro ET, Richmond JC, Rockett SE, McGrath MM, Donaldson WR. The use of a generic, patient-based health assessment (SF-36) for evaluation of patients with anterior cruciate ligament injuries. Am J Sports Med. 1996 Mar-Apr;24(2): 196-200.

24. Paxton ES, Kymes SM, Brophy RH. Cost-effectiveness of anterior cruciate ligament reconstruction: a preliminary comparison of single-bundle and doublebundle techniques. Am J Sports Med. 2010 Dec;38(12):2417-25. Epub 2010 Sep 9.

25. Lubowitz JH, Appleby D. Cost-effectiveness analysis of the most common orthopaedic surgery procedures: knee arthroscopy and knee anterior cruciate ligament reconstruction. Arthroscopy. 2011 Oct;27(10):1317-22. Epub 2011 Aug 19.

26. Gottlob CA, Baker CL Jr, Pellissier JM, Colvin L. Cost effectiveness of anterior cruciate ligament reconstruction in young adults. Clin Orthop Relat Res. 1999 Oct; (367):272-82.

27. Genuario JW, Faucett SC, Boublik M, Schlegel TF. A cost-effectiveness analysis comparing 3 anterior cruciate ligament graft types: bone-patellar tendon-bone autograft, hamstring autograft, and allograft. Am J Sports Med. 2012 Feb;40(2):30714. Epub 2011 Nov 15.

28. Farshad M, Gerber C, Meyer DC, Schwab A, Blank PR, Szucs T. Reconstruction versus conservative treatment after rupture of the anterior cruciate ligament: cost effectiveness analysis. BMC Health Serv Res. 2011 Nov 19;11:317.

29. Vitale MA, Vitale MG, Zivin JG, Braman JP, Bigliani LU, Flatow EL. Rotator cuff repair: an analysis of utility scores and cost-effectiveness. J Shoulder Elbow Surg. 2007 Mar-Apr;16(2):181-7.

30. Räsänen $P$, Paavolainen $P$, Sintonen $H$, Koivisto $A M$, Blom $M$, Ryynänen $O P$, Roine RP. Effectiveness of hip or knee replacement surgery in terms of qualityadjusted life years and costs. Acta Orthop. 2007 Feb;78(1):108-15.

31. Al-Ruzzeh S, Epstein D, George S, Bustami M, Wray J, Ilsley C, Sculpher M, Amrani M. Economic evaluation of coronary artery bypass grafting surgery with and without cardiopulmonary bypass: cost-effectiveness and quality-adjusted life years in a randomized controlled trial. Artif Organs. 2008 Nov;32(11):891-7.

32. Orfaly RM, McConkey JP, Regan WD. The fate of meniscal tears after anterior cruciate ligament reconstruction. Clin J Sport Med. 1998 Apr;8(2):102-5.

33. Harrell FE Jr. Regression modeling strategies: with applications to linear models, logistic regression, and survival analysis. New York: Springer; 2010. 34. Miller ME, Hui SL, Tierney WM. Validation techniques for logistic regression models. Stat Med. 1991 Aug;10(8):1213-26. 